\title{
OFERTA Y DEMANDA DE CAMPOS DE PRÁCTICA CLÍNICA PARA LA FORMACIÓN DE PREGRADO DE ESTUDIANTES DE CIENCIAS DE LA SALUD EN EL PERÚ, 2005-2009
}

\author{
Javier Alva ${ }^{1, a}$, George Verastegui,a, Edgar Velasquez ${ }^{3, b}$, \\ Reyna Pastor ${ }^{3, \mathrm{~b}}$, Betsy Moscoso $0^{3, a}$
}

\begin{abstract}
RESUMEN
Objetivos. Describir la oferta y demanda de campos clínicos para los estudiantes de pregrado del Perú. Materiales y métodos. Se realizó un estudio descriptivo, se consideró como oferta de campos clínicos al total de camas hospitalarias existentes en el Perú. La demanda fue calculada con el número total de alumnos matriculados en las carreras de ciencias de la salud que cursan años clínicos o internado. Se calculó el número de camas por alumno y la cobertura del campo clínico tanto a nivel nacional como en algunas regiones seleccionadas (Lima, Arequipa, La Libertad y Lambayeque). Resultados. Para el 2009 el Perú tiene 34539 camas hospitalarias, 78,5\% pertenecen al sector público y 48,4\% están ubicadas en Lima. Para el año 2008 se estimó que 44032 alumnos requirieron de campos clínicos, 70\% provenían de universidades privadas, las cuales crecieron $65 \%$ desde el 2005 . La cobertura de campos clínicos, solo considerando a internos de cuatro carreras (medicina, enfermería, obstetricia y odontología), llega al 31,5\% a nivel nacional. El número de camas por estudiante osciló entre 0,5 en La Libertad a 0,82 en Lima siendo el promedio nacional de 0,45. Conclusiones. La oferta de campos clínicos para la enseñanza de pregrado en el Perú es insuficiente para satisfacer la demanda, que continúa en crecimiento a expensas de universidades privadas, por lo que se requiere con urgencia de una regulación.
\end{abstract}

Palabras clave: Educación médica; Estudiantes del área de la salud; Hospitales; Política de Salud; Perú (fuente: DeCS BIREME).

\section{SUPPLY AND DEMAND OF CLINICAL PRACTICE FIELDS FOR TRAINING UNDERGRADUATE HEALTH SCIENCES STUDENTS IN PERU, 2005-2009}

\begin{abstract}
Objectives: To describe the supply and demand of clinical fields for undergraduate students of Peru. Materials and methods. A descriptive study was considering as supply of clinical fields the total number of existing hospital beds in Peru. The demand was calculated using the total number of alumni registered in health science carrers following the clinical years or the internship. We calculated the number of beds per student and the coverage of clinical fields nationally and in some selected regions (Lima, Arequipa, La Libertad and Lambayeque). Results. In 2009, Peru had 34,539 hospital beds, $78.5 \%$ of which pertained to the public sector and $48.4 \%$ are from Lima. We estimated that in 2008 44,032 alumni needed clinical fields, $70 \%$ from private universities, which grew $65 \%$ since 2005 . The coverage of clinical fields, considering only interns from four carreers (medicine, nursery, obstetrics and dentistry) was only $31.5 \%$ at the national level. The number of beds per student oscillated between 0.5 in La Libertad to 0.82 in Lima with a national mean of 0.45 . Conclusions. The supply of clinical fields for teaching undergraduates is insufficient to satisfy the demand, which continues to grow because of private universities, and hence requires urgent regulation.
\end{abstract}

Key words: Education, medical; Students, health occupations; Hospitals; Health policies; Peru (source: MeSH NLM).

\footnotetext{
Sistema Metropolitano de Solidaridad (SISOL). Lima, Perú.

Gerencia ACESALUD. Lima, Perú.

3 Observatorio de Recursos Humanos, Dirección General de Gestión del Desarrollo de Recursos Humanos, Ministerio de Salud. Lima, Perú.

a Médico; ${ }^{b}$ Estadístico.
} 


\section{INTRODUCCIÓN}

Para la formación de profesionales en salud es indudable la necesidad del contacto con el paciente que, por lo general, se da en los cursos clínicos e internado que les permite adquirir habilidades clínicas ${ }^{(1-3)}$; incluso existen escuelas que propugnan la exposición temprana a los pacientes en los primeros años, para mejorar la comprensión y rendimiento de los estudiantes en los cursos preclínicos ${ }^{(4,5)}$.

Los campos de formación de los profesionales de la salud, también llamados campos clínicos, se ubican en los servicios de salud ${ }^{(6)}$, situación que en el Perú se da desde el Virreinato ${ }^{(1)}$, desde entonces, los procesos de aprendizaje de los médicos y otros profesionales de la salud se han concentrado en los grandes centros asistenciales ${ }^{(2,7-9)}$

Los servicios de salud hospitalarios han devenido en el escenario en el que se ubican los campos de formación ${ }^{(10)}$, donde el modelo sanitario tradicional de producción de servicios y de formación de recursos humanos de salud, privilegia a los servicios hospitalarios de mediana y alta complejidad como el escenario central de desarrollo y asiento de los campos clínicos.

Debido a que el proceso formativo en los campos clínicos depende del acceso a los pacientes ambulatorios u hospitalizados, se generan situaciones que deben ser tomadas en cuenta, como el deseo del paciente de ser evaluado por alumnos, la cantidad de alumnos que pueden examinarlos, entre otras ${ }^{(11,12)}$, lo que ha ido generando regulaciones para evitar una saturación o incomodidad hacia el paciente, entre ellas se han generado indicadores como el número de camas hospitalarias por alumno para determinar el máximo de estudiantes que pueden acceder a un hospital como campo clínico ${ }^{(6,13-15)}$.

En el Perú, como en otros países latinoamericanos, se ha visto un incremento en el número de facultades y alumnos de ciencias de la salud ${ }^{(16,17)}$, lo cual incrementa la demanda por campos clínicos y, además, una sobrepoblación de estudiantes en los campos clínicos ${ }^{(18)}$. Por ello, el estudio tuvo como objetivo describir la oferta y demanda de campos clínicos para los estudiantes de pregrado del Perú durante el año 2008.

\section{MATERIALES Y MÉTODOS}

Se realizó un estudio descriptivo entre julio y diciembre de 2010, para ello se usaron fuentes de información secundarias. El estudio fue revisado y aprobado por la
Dirección General de Gestión del Desarrollo de Recursos Humanos del Ministerio de Salud del Perú.

Se consideró como oferta de campos clínicos al total de camas hospitalarias existentes en el Perú, para lo cual se realizó una revisión de la base de datos de establecimientos de salud que tiene la Oficina General de Estadística e Informática del Ministerio de Salud actualizada para el periodo 2005 al 2009. Esta información se desagregó por regiones y subsectores: MINSA, EsSalud (Seguridad Social), sanidades (incluye fuerzas policiales y militares) y privados.

Para la identificación de la demanda de campos de formación, se consideró a las 13 carreras profesionales vinculadas con la salud: medicina humana, enfermería, obstetricia, odontología (estomatología), farmacia y bioquímica, nutrición, tecnología médica, psicología, biología (o ciencias biológicas), medicina veterinaria, trabajo social, ingeniería sanitaria y administración en salud; sin embargo, solo se incluyo a diez de ellas por no tener acceso a información de tres (trabajo social, ingeniería sanitaria y administración en salud).

Se usó la base de datos de alumnos matriculados por año de la Asamblea Nacional de Rectores durante el periodo 2005-2009. La información sobre la matrícula anual, engloba a todas las promociones de una carrera que se encuentran estudiando en un determinado año. Para establecer el número de alumnos matriculados de una promoción anual, se ponderó el total de matriculados entre el número de años de la carrera, diferenciados para medicina (siete años) y el resto de carreras (cinco años) para obtener el número promocional de estudiantes por carrera.

Para estimar la demanda potencial de campos de formación, se calculó, en primer lugar, el total de alumnos que potencialmente harán uso de las instalaciones hospitalarias, de todas las carreras profesionales solo se consideró para este cálculo las carreras de medicina humana, enfermería, obstetricia y odontología. Los alumnos de estas carreras fueron divididos en internos (último año de la carrera) y alumnos de clínicas (aquellos que pasan mayor parte de su tiempo de formación en un hospital) que para la carrera de medicina se consideró tres años y para las otras dos años. Luego se realizó el cálculo de campos clínicos necesarios, para lo cual se multiplicó el número de alumnos que potencialmente harán el uso de instalaciones hospitalarias por cinco, que es el número mínimo de camas que deben existir por alumno o interno ${ }^{(13)}$.

Se realizó la evaluación tanto a nivel nacional como en cuatro regiones seleccionadas por ser las que concentran el mayor volumen de demanda potencial de campos 
de formación por la presencia de universidades públicas y privadas, estas fueron Lima, Arequipa, La Libertad y Lambayeque.

Finalmente, se estimó el número de camas por alumno, que hay disponibles tanto a nivel nacional como regional, según carrera o si estaban en internado o años clínicos, para ello se realizaron dos cálculos. El primero, tomando en cuenta que la totalidad de camas disponibles, tanto a nivel público como privado, se encuentran disponibles para la enseñanza de alumnos de pregrado.

Se realizó un segundo cálculo, en el cual se estimó el número de camas realmente disponibles para la enseñanza del pregrado, para lo cual se ajustó por dos factores:

Uso restringido de la total capacidad de los campos clínicos privados. Son escasas las clínicas privadas que están disponibles para la enseñanza del pregrado; además, las universidades públicas y privadas en el Perú tienen como principal fuente de campos clínicos los hospitales públicos; no tienen clínicas docentes particulares para la enseñanza de la medicina, pero sí en algunos casos para odontología, obstetricia y enfermería ${ }^{(13)}$. Se ajustó el número de camas de hospitales privados por el factor 0,3 , este valor fue decidido en forma arbitraria por considerar que solo el $30 \%$ de los campos clínicos privados están disponibles para la formación profesional.

No disponibilidad de la totalidad del campo clínico instalado. No todos los servicios de un hospital aceptan alumnos regularmente para la enseñanza del pregrado, no en todas las regiones del país o ciudades donde hay hospitales existe formación de estudiantes de ciencias de la salud, adicionalmente, no todos los pacientes están en capacidad de aceptar participar como sujetos de estudio por estudiantes, ya sea por una condición de salud muy delicada o su negativa al examen o anamnesis ${ }^{(11,12)}$. Por ello se ajustó el total de camas por el factor 0,7 , que sería una aproximación al porcentaje de camas realmente disponibles.

Teniendo en cuenta estas premisas se usó la siguiente fórmula para estimar el número de camas disponibles: [(camas privadas * 0,3) + camas públicas] * 0,7. Los datos fueron procesados utilizando el programa Excel 2007.

\section{RESULTADOS}

\section{LA OFERTA DE CAMPOS CLÍNICOS}

Para el año 2009, se registran 469 hospitales en el Perú, 155 (33\%) son del MINSA, 79 (17\%) de EsSalud, $18(4 \%)$ a las sanidades de las Fuerzas Armadas y
Policiales y $217(46 \%)$ al sector privado. Un tercio del total de hospitales del país, se concentran en Lima (34\%) y sumado a cuatro regiones concentran más de la mitad de los hospitales del país ( $8 \%$ en la Libertad, $6 \%$ en Piura y $5 \%$ en Ancash).

Para el periodo 2005-2009, se evidencia un crecimiento global del número de hospitales de alrededor del $5 \%$ (de 448 a 469), el MINSA pasó de 147 a 155 hospitales ( $5,4 \%$ de crecimiento), EsSalud se mantuvo en el mismo número de hospitales (79), las Sanidades disminuyeron de 21 a $18(-14,3 \%)$ y el sector privado tuvo un incremento de 201 a 217 (8,0\%).

El total de camas hospitalarias del sector salud registradas para el año 2009 fue de 34539 camas, 17436 $(50,5 \%)$ en el MINSA, 6753 en EsSalud (19,6\%), 2907 en las Sanidades $(8,4 \%)$ y 7443 en el sector privado $(21,5 \%)$. Lima concentra casi la mitad $(48,4 \%)$ del total nacional de camas hospitalarias, que junto a las regiones La Libertad, Callao y Arequipa (cada una de ellas con el $5 \%$ ) concentran el $63 \%$ del total de camas existentes en el país (Tabla 1).

Para el periodo 2005-2009 el incremento global llegó al $12 \%$. El MINSA tuvo un incremento de $11 \%$ ( $2 \%$ anual), EsSalud no registró crecimiento, las Sanidades y el sector privado crecieron cada uno alrededor del $25 \%$ ( $5 \%$ anual).

\section{LAS CARRERAS UNIVERSITARIAS DE SALUD EN EL PERÚ}

En el 2008 estaban matriculados 120502 alumnos en las diez carreras de ciencias de la salud estudiadas, las cuatro carreras que requieren de mayores recursos de campos clínicos (medicina, enfermería, obstetricia y odontología) acumulan casi dos tercios del total de matriculados en carreras de ciencias de la salud (75 608 alumnos, 64\%).

Las carreras que registran el mayor número de alumnos matriculados son enfermería (26 698), odontología (20 626), medicina (17 700), psicología (17 363) y obstetricia (10 584), a predominio de las universidades privadas que concentran el $65,85,59$ y $68 \%$ de estudiantes, respectivamente. Las universidades privadas incrementaron el número de estudiantes matriculados del 2005 al 2008 en 63\% en promedio (Tabla 2).

En el caso de medicina, cinco universidades representan el $82 \%$ del total de matriculados en universidades privadas y una de ellas tiene el $30 \%$ del total. En enfermería, once universidades concentran el $87 \%$ de matriculados y dos de ellas tienen el $45 \%$ del total. Para obstetricia, 
Tabla 1. Oferta de campos clínicos en el Perú y regiones seleccionadas según proveedor, 2009.

\begin{tabular}{|c|c|c|c|c|c|}
\hline \multirow{2}{*}{ Región } & MINSA & EsSalud & Sanidades & Privados & Total \\
\hline & n (\%) & n (\%) & n (\%) & n (\%) & $\mathbf{N}(\%)$ \\
\hline \multicolumn{6}{|l|}{ Hospitales } \\
\hline Lima & $30(19,4)$ & $14(17,7)$ & $7(38,9)$ & $107(49,3)$ & $158(33,7)$ \\
\hline La Libertad & $10(6,5)$ & $5(6,3)$ & $0(0,0)$ & $22(10,1)$ & $37(7,9)$ \\
\hline Lambayeque & $3(1,9)$ & $3(3,8)$ & $1(5,6)$ & $14(6,5)$ & $21(4,5)$ \\
\hline Arequipa & $5(3,2)$ & $5(6,3)$ & $3(16,7)$ & $5(2,3)$ & $18(3,8)$ \\
\hline Resto del país & $107(69,0)$ & $52(65,8)$ & $7(38,9)$ & $69(31,8)$ & $235(50,1)$ \\
\hline Total & $155(33,0)$ & $79(16,8)$ & $18(3,8)$ & $217(46,3)$ & $469(100,0)$ \\
\hline \multicolumn{6}{|l|}{ Camas } \\
\hline Lima & $7411(42,5)$ & $3002(44,5)$ & $1827(62,8)$ & $4462(59,9)$ & $16702(48,4)$ \\
\hline La Libertad & $794(4,6)$ & $321(4,8)$ & $0(0,0)$ & $651(8,7)$ & $1766(5,1)$ \\
\hline Lambayeque & $324(1,9)$ & $436(6,5)$ & $82(2,8)$ & $348(4,7)$ & $1190(3,4)$ \\
\hline Arequipa & $1021(5,9)$ & $484(7,2)$ & $98(3,4)$ & $118(1,6)$ & $1721(5,0)$ \\
\hline Resto del país & $7886(45,2)$ & $2510(37,2)$ & $900(31,0)$ & $1864(25,0)$ & $13160(38,1)$ \\
\hline Total & $17436(50,5)$ & $6753(19,6)$ & $2907(8,4)$ & $7443(21,5)$ & $34539(100,0)$ \\
\hline
\end{tabular}

diez universidades tienen el $63 \%$ de matriculados y tres de estas tienen el $57 \%$ del total. En odontología, cuatro universidades concentran el $65 \%$ de matriculados y una de ellas tiene el $31 \%$ del total.

En el periodo 2005-2008, en el sector privado las carreras que más incrementaron en números absolutos fueron odontología (8 644 alumnos), enfermería (7 146), psicología (4 422) y medicina (3 047), en el sector públi$\mathrm{co}$, las carreras que tuvieron mayor incremento fueron biología (1 213), tecnología médica (1 064) y psicología (945).

\section{LA DEMANDA POTENCIAL DE CAMPOS DE FORMACIÓN PARA EL 2008}

En el año 2008 se estima que 44932 alumnos requirieron de campos clínicos para su formación en el Perú, tanto a nivel de internado como año de clínicas, de los cuales 13618 son de universidades públicas (30\%) y 31314 de universidades privadas (70\%).
Entre los años 2005 y 2008 la demanda de campos de formación se incrementó en un 43\%; el número de alumnos en clínicas e internado pasó de 31407 a 44932. En este periodo, el incremento se produce de manera diferenciada, mientras en las universidades públicas fue de $10 \%$ (de 12415 a 13 618), en las universidades privadas fue de $65 \%$ (de 18992 a 31314 ).

Los estudiantes de medicina que demandan campos clínicos representan el $22,5 \%$ del total de estudiantes de ciencias de la salud en esa condición a nivel nacional, estos porcentajes cambian según región, Lima $(23,2 \%)$, Lambayeque (30,5\%), Arequipa (49,2\%) y La Libertad $(53,4 \%)$. Los datos específicos de la cantidad de alumnos según carrera, región y nivel de estudios se pueden apreciar en la Tabla 3.

Al calcular la cobertura de campos clínicos considerando que se necesitan por lo menos cinco camas por estudiante, se encontró que a nivel nacional solo se alcanza el $68,3 \%$ de la demanda si consideramos únicamente

Tabla 2. Alumnos de pregrado de ciencias de la salud matriculados en el año 2005 y 2008 en el Perú e incremento.

\begin{tabular}{|c|c|c|c|c|c|c|c|c|c|}
\hline \multirow{2}{*}{ Carrera profesional } & \multicolumn{3}{|c|}{2008} & \multicolumn{3}{|c|}{2005} & \multicolumn{3}{|c|}{ Incremento 2008-2005 (\%) } \\
\hline & Total & Privadas & $(\%)$ & Total & Privadas & $(\%)$ & Total & Privadas & Públicas \\
\hline Enfermería & 26698 & 17324 & $(64,9)$ & 18898 & 10178 & $(53,9)$ & 41,3 & 70,2 & 7,5 \\
\hline Odontología & 20626 & 17634 & $(85,5)$ & 11535 & 8990 & $(77,9)$ & 78,8 & 96,2 & 17,6 \\
\hline Medicina & 17700 & 10495 & $(59,3)$ & 13982 & 7448 & $(53,3)$ & 26,6 & 40,9 & 10,3 \\
\hline Psicología & 17363 & 14164 & $(81,6)$ & 11976 & 9722 & $(81,2)$ & 45,0 & 45,7 & 41,9 \\
\hline Obstetricia & 10584 & 7229 & $(68,3)$ & 8854 & 5387 & $(60,8)$ & 19,5 & 34,2 & $-3,2$ \\
\hline Farmacia y bioquímica & 7286 & 4429 & $(60,8)$ & 4892 & 2679 & $(54,8)$ & 48,9 & 65,3 & 29,1 \\
\hline Biología & 6648 & 1543 & $(23,2)$ & 4345 & 453 & $(10,4)$ & 53,0 & 240,6 & 31,2 \\
\hline Medicina veterinaria & 6098 & 2535 & $(41,6)$ & 5175 & 2004 & $(38,7)$ & 17,8 & 26,5 & 12,4 \\
\hline Tecnología médica & 4974 & 3339 & $(67,1)$ & 2048 & 1477 & $(72,1)$ & 142,9 & 126,1 & 186,3 \\
\hline Nutrición & 2525 & 790 & $(31,3)$ & 1624 & 438 & $(27,0)$ & 55,5 & 80,4 & 46,3 \\
\hline Total & 120502 & 79482 & $(66,0)$ & 83329 & 48776 & $(58,5)$ & 44,6 & 63,0 & 18,7 \\
\hline
\end{tabular}


Tabla 3. Estudiantes de ciencias de la salud que requieren de campos clínicos de formación a nivel nacional y en regiones seleccionadas por tipo de universidad y carrera profesional, Perú 2008.

\begin{tabular}{|c|c|c|c|c|c|c|c|c|c|c|}
\hline \multirow{2}{*}{$\begin{array}{l}\text { Universidad } \\
\text { Carreras }\end{array}$} & \multicolumn{2}{|c|}{ Nacional } & \multicolumn{2}{|c|}{ Lima } & \multicolumn{2}{|c|}{ La Libertad } & \multicolumn{2}{|c|}{ Lambayeque } & \multicolumn{2}{|c|}{ Arequipa } \\
\hline & Internado & Clínica & Internado & Clínica & Internado & Clínica & Internado & Clínica & Internado & Clínica \\
\hline \multicolumn{11}{|l|}{ Pública } \\
\hline Medicina & 1030 & 3090 & 268 & 804 & 96 & 288 & 54 & 162 & 116 & 348 \\
\hline Enfermería & 1875 & 3750 & 150 & 300 & 73 & 146 & 68 & 136 & 111 & 222 \\
\hline Obstetricia & 692 & 1384 & 114 & 228 & 0 & 0 & 0 & 0 & 0 & 0 \\
\hline Odontología & 599 & 1198 & 193 & 386 & 29 & 58 & 0 & 0 & 0 & 0 \\
\hline Sub-Total & 4196 & 9422 & 725 & 1718 & 198 & 492 & 122 & 298 & 227 & 570 \\
\hline \multicolumn{11}{|l|}{ Privada } \\
\hline Medicina & 1500 & 4500 & 828 & 2824 & 371 & 1113 & 69 & 207 & 156 & 468 \\
\hline Enfermería & 3465 & 6930 & 1622 & 3244 & 226 & 452 & 186 & 372 & 69 & 138 \\
\hline Obstetricia & 1446 & 2892 & 615 & 1230 & 90 & 180 & 119 & 238 & 45 & 90 \\
\hline Odontología & 3527 & 7054 & 2513 & 5026 & 126 & 252 & 0 & 0 & 149 & 298 \\
\hline Subtotal & 9938 & 21376 & 5578 & 12324 & 813 & 1997 & 374 & 817 & 419 & 994 \\
\hline Total & 14134 & 30798 & 6303 & 14042 & 1011 & 2431 & 496 & 1115 & 646 & 1564 \\
\hline
\end{tabular}

a los estudiantes de medicina, $31,5 \%$ si consideramos a los internos de medicina, obstetricia, enfermería y odontología y sería del $15,4 \%$ si se considera a todos los estudiantes (incluyendo internos) de esas cuatro carreras que requieren de campos clínicos. La región que tiene una menor cobertura, considerando el total de estudiantes es La Libertad con $10,3 \%$ y la mayor Lima con $16,4 \%$. Para el caso de internos es Arequipa la que tiene menor cobertura con $29,2 \%$, pero en el caso de estudiantes de medicina La Libertad tiene una escasa cobertura llegando solo al 18,9\% (Figura 1).

Considerando la cobertura solo en función de estudiantes de medicina y que se usa la totalidad de las camas de hospitales públicos y privados, existe un déficit de

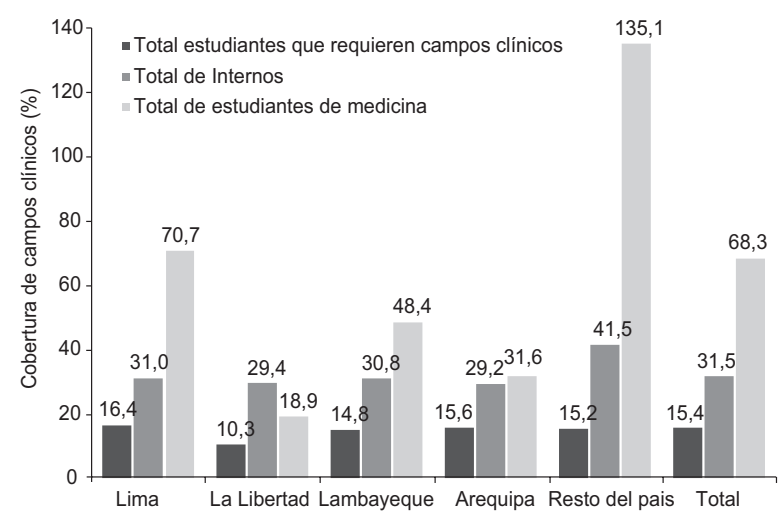

Figura 1. Cobertura de campos clínicos en el Perú a nivel nacional y regiones seleccionadas, 2008.

Para el cálculo de la cobertura se consideró el total de camas a nivel nacional o región entre la demanda (número de estudiantes por 5). campos clínicos de 16061 a nivel nacional, 7574 en La Libertad, 6918 en Lima, 3719 en Arequipa y 1270 en Lambayeque. Si se ajustara estos datos por el número real de camas disponibles o por el número total de estudiantes que requieren de campos clínicos (medicina, odontología, obstetricia y medicina), el déficit sería aun mayor.

Cuando se realizó el cálculo de número de camas por alumno considerando el total de estudiantes que demandan campos clínicos, el valor osciló entre 0,5 en La Libertad a 0,82 en Lima, siendo el promedio nacional de 0,45, pero el estándar internacional es de 5,0 (Tabla 4), estos valores fueron considerando que todas las camas son accesibles, cuando se realizó la estimación de las camas disponibles estos valores fueron inferiores. Aun considerando solo a los estudiantes de medicina, en ninguna de las regiones estos valores fueron mayores a cinco (Tabla 4).

\section{DISCUSIÓN}

Nuestros resultados corroboran la situación de grave desequilibrio entre la oferta y la demanda de campos de formación en el Perú, situación ya expuesta en el 2007, y que ha empeorado en lugar de mejorar ${ }^{(13)}$. Este desequilibrio ha sido propiciado por un incremento en la demanda por parte de las universidades privadas, que en el Perú, al menos para el caso de medicina, no tienen hospitales docentes propios y hacen uso extensivo de los establecimientos públicos del Ministerio de Salud, EsSalud y Sanidades.

En otros países de Latinoamérica, hay escuelas de medicina privadas que tienen hospitales docentes propios 
Tabla 4. Número de camas por estudiantes que demandan campos clínicos según región y carrera profesional considerando la totalidad de camas y el estimado de camas disponibles, Perú 2008.

\begin{tabular}{|c|c|c|c|c|c|}
\hline Carreras & Nacional & Lima & La Libertad & Lambayeque & Arequipa \\
\hline \multicolumn{6}{|c|}{ Asumiendo la totalidad de camas existentes* } \\
\hline Medicina & 1,91 & 3,54 & 0,95 & 2,42 & 1,58 \\
\hline Enfermería & 1,26 & 3,14 & 1,97 & 1,56 & 3,19 \\
\hline Obstetricia & 2,99 & 7,64 & 6,54 & 3,33 & 12,75 \\
\hline Odontología & 1,79 & 2,06 & 3,80 & & 3,85 \\
\hline Total & 0,45 & 0,82 & 0,50 & 0,74 & 0,78 \\
\hline \multicolumn{6}{|c|}{ Ajustado a la capacidad de camas disponibles** } \\
\hline Medicina & 1,13 & 2,01 & 0,49 & 1,35 & 1,05 \\
\hline Enfermería & 0,75 & 1,79 & 1,02 & 0,87 & 2,12 \\
\hline Obstetricia & 1,77 & 4,35 & 3,40 & 1,85 & 8,50 \\
\hline Odontología & 1,06 & 1,17 & 1,97 & & 2,57 \\
\hline Total & 0,27 & 0,47 & 0,26 & 0,41 & 0,52 \\
\hline
\end{tabular}

* Se consideró la máxima capacidad instalada de camas hospitalarias tanto en el sector público como privado.

** Se estimó el número de camas disponibles usando la siguiente fórmula: [(camas privadas * 0,3$)+$ camas públicas] * 0,7 .

o convenios con establecimientos privados ${ }^{(19,20)}$, de esa forma aseguran a sus alumnos los campos clínicos sin entrar en conflicto con otras universidades.

Esta situación lleva a la reflexión sobre quién es el dueño del campo clínico público y qué consideraciones se deben tomar en cuenta para su regulación en el país. Es indudable que el más afectado resulta ser el paciente del hospital público pues debe ser examinado en múltiples rondas por alumnos de diferentes carreras y facultades a la vez o en forma consecutiva; pero, también se afecta el proceso de formación del profesional de la salud, en el que las oportunidades de una mejor interacción con el paciente se ven reducidas por la gran cantidad de alumnos ${ }^{(18,21)}$.

Cada institución de salud tiene sus procedimientos para el acceso al campo clínico, por lo tanto,es necesario una normativa general y la acreditación de los centros que estén en capacidad de recibir alumnos, teniendo en cuenta la infraestructura, docentes y, sobre todo, el bienestar de los pacientes. Así mismo, queda la duda del por qué el Estado, a través de los establecimientos de salud del Ministerio de Salud, EsSalud y Sanidades, deben subsidiar la educación privada. Teniendo en cuenta que existe un pequeño grupo de universidades de gestión privada que concentran la mayoría de los estudiantes de las carreras de salud cuyo comportamiento expandiendo ilimitadamente la demanda escapa una racionalidad técnica.

Cuando se evaluó la cobertura de la demanda de campos clínicos considerando a cinco camas por alumno como estándar, se encontró una pobre cobertura, tanto a nivel nacional como en las regiones seleccionadas, esto considerando que toda la capacidad instalada (todas las camas de los establecimientos de salud, públicos y privados) son utilizados como campos clínicos; sin embargo, si tenemos en cuenta que en el Perú son escasos los hospitales privados donde se da la enseñanza de estudiantes de pregrado y que no todas las camas están disponibles para los alumnos, el número de camas por alumno oscila entre 1,26 y 2,99 a nivel nacional, según la carrera evaluada.

Esto puede ser un indicador de una improvisación de la educación en salud, ya que se requiere la planificación del equipo docente, infraestructura y del acceso al campo clínico. Por ejemplo, el Examen Nacional de Medicina (ENAM) que se toma a todos los internos de medicina peruanos en forma obligatoria desde el $2007^{(22)}$, muestra que el $36,8 \%$ de los postulantes entre el 2007 y 2009 lo reprobaron ${ }^{(23)}$.

El estudio presenta ciertas limitaciones, ya que se ha incluido en la oferta de campos clínicos a todos los establecimientos de salud, públicos o privados, sin saber con precisión cuáles son los que son utilizados para la enseñanza de pregrado, ya que en varias regiones no existen facultades de ciencias de la salud o, como ya se mencionó, los establecimientos privados no abren sus puertas a la enseñanza universitaria. Al usarse fuentes de información secundaria, aunque son los datos oficiales, puede ser que algunos no se ajusten totalmente a lo que esté sucediendo, sin embargo, no hemos encontrado una mejor forma de tener acceso a dicha información. Por otro lado, en estos últimos años (2010-2011) se han construido nuevos hospitales en Lima y en regiones tanto del MINSA como EsSalud que estarían incrementado la oferta de campos clínicos y no han sido incluidos en este análisis por no tener aún la confirmación del número de camas disponibles y no estar operativos para el 
periodo de estudio. Además en el caso de Lima hay que tener en cuenta que varias universidades de provincia hacen rotaciones o el internado en hospitales de Lima variable que no fue considerada en este análisis.

Adicionalmente, cabe precisar que por el limitado acceso a la información no se pudo diferenciar el tipo de cama, ya que existen camas hospitalarias, de urgencias, de cuidados intensivos, obstétricas, entre otras; por ese motivo se realizaron los ajustes con los factores mencionados en la metodología del estudio.

Así mismo, la definición de campo clínico que hemos trabajado hace expresa alusión al número de camas hospitalarias; sin embargo, no es el único escenario donde se puede realizar la formación de los profesionales de la salud, más aun si consideramos que estos deben formarse para las necesidades del país que debe privilegiar el primer nivel de atención, donde la labor es en centros de salud y con la comunidad y no en el hospital.

Se requieren futuros estudios que aborden con mayor amplitud el concepto de campo clínico o espacio de formación, que evalúe en forma diferenciada el tipo de cama hospitalaria, el nivel del establecimiento de salud (I, II o III nivel), identificar a los establecimientos de salud que son usados actualmente como campos clínicos, evaluar número de docentes por hospital y consultorios habilitados para la docencia.

Esta aproximación inicial, a pesar de las limitaciones, es importante porque nos muestra la brecha entre la oferta y la demanda de campos clínicos en el Perú y la urgente necesidad de su regulación. Por ello, se propone que el MINSA sin menoscabo de su participación en los espacios de articulación docencia-servicio defina y tome iniciativas en correspondencia con el hecho de ser el mayor contribuyente de la oferta de campos de formación del país, así como ser el principal empleador de los recursos humanos en salud.

En conclusión, la oferta de campos clínicos para la enseñanza de pregrado en el Perú es insuficiente para satisfacer la demanda, que continúa en crecimiento a expensas de universidades privadas, por lo que se requiere, con urgencia de una regulación en beneficio tanto de los estudiantes en formación como de los pacientes, quienes también participan en el proceso de aprendizaje con su situación de salud.

\section{AGRADECIMIENTOS}

Este estudio ha sido posible por el apoyo e interés en promover la investigación en recursos humanos en salud en el país, del equipo de la Dirección General de Gestión del Desarrollo de Recursos Humanos en Salud (DGDRH) del MINSA, encabezados por su Director Dr. Manuel Núñez. Igualmente ha sido valiosa la colaboración del Dr. Percy Mayta-Tristán, quien tuvo la paciencia de analizar y discutir los contenidos del estudio, brindando valiosas apreciaciones que han enriquecido el artículo.

\section{Contribuciones de Autoría}

JA, RP y BM generaron la idea de investigación y el diseño del estudio, JA, GV y EV realizaron la recolección y análisis de datos, BM y RP supervisaron la ejecución del estudio, JA elaboró el manuscrito y GV; EV, RP y BM aportaron críticamente a su contenido, todos los autores aprobaron la versión final a publicar.

\section{Fuentes de Financiamiento}

Dirección General de Gestión del Desarrollo de los Recursos Humanos, Ministerio de Salud, Lima, Perú

\section{Conflictos de Interés}

Los autores declaran no tener conflictos de interés en la publicación de este artículo.

\section{REFERENCIAS BIBLIOGRÁFICAS}

1. Rabí M. La formación de médicos y cirujanos durante los siglos XVI a XIX: las escuelas prácticas de medicina y cirugía en el Perú. An Fac Med (Lima). 2006;67(2):173-83.

2. Lifghitz A. La enseñanza de la competencia clínica. Gac Med Mex. 2004;3(140):312-3.

3. Lurie SJ, Mooney CJ. Relationship between clinical assessment and examination scores in determining clerkship grade. Med Educ. 2010;44(2):177-83.

4. Ottenheijm RP, Zwietering PJ, Scherpbie AJ, Metsemakers JF. Early student-patient contacts in general practice: an approach based on educational principles. Med Teach. 2008;30(8):802-8.

5. Littlewood S, Ypinazar V, Margolis SA, Scherpbier A, Spencer J, Dornan T. Early practical experience and the social responsiveness of clinical education: systematic review. BMJ. 331:387-91.

6. Secretaría de Salud de México. Norma Oficial Mexicana NOM-234-SSA1-2003. Utilización de campos clínicos para ciclos clínicos e internado de pregrado. México DF: Secretaría de Salud; 2003.

7. Papastavrou E, Lambrinou E, Tsangari H, Saarikoski M, Leino-Kilpi H. Student nurses experience of learning in the clinical environment. Nurse Educ Pract. 2010;10(3):17682.

8. Raisler J, O'Grady M, Lori J. Clinical teaching and learning in midwifery and women's health. J Midwifery Womens Health. 2003;48(6):398-406.

9. Stewart CJ, Moloney EJ, Kinirons MJ. Clinical experiences of undergraduate dental students in pediatric dentistry at Cork University Dental School and Hospital, Ireland. J Dent Educ. 2010;74(3):325-30. 
10. Ferreira JR. Misión del hospital a la luz de las nuevas tendencias de la educación médica. Educ Med Salud. 1976;10(2):140-51.

11. Olson LG, Hill SR, Newby DA. Barriers to student access to patients in a group of teaching hospitals. Med J Austr. 2005;183(9):461-3.

12. Cooke F, Galasko G, Ramrakha V, Richards D, Rose A, Watkins J. Medical students in general practice: how do patients feel? Br J Gen Practice. 1996;46:361-2.

13. Arroyo J. Análisis y propuesta de criterios de acreditación de campos clínicos de práctica en la formación de pre y postgrado de los profesionales de la salud. Lima: MINSA; 2007.

14. Chile, Ministerio de Salud. Norma general técnica y administrativa $\mathrm{N}^{\circ} 18$ sobre asignación y uso de los campos de formación profesional y técnica en el sistema nacional de servicios de salud y normas de protección para sus funcionarios, académicos, estudiantes y usuarios. Santiago de Chile: Ministerio de Salud; 2010.

15. Colombia, Ministerio de Salud. Un nuevo paradigma en la relación entre la formación y los servicios de salud. Propuesta de estándares para la acreditación de los centros de formación en salud. Bogotá: Ministerio de Salud; 2002.

16. Arroyo J. Los sistemas descentrados de recursos humanos en salud: el caso del Perú, 1990-2005. Ciencia Saude Colectiva. 2006;11(4):1063-72.

17. Goic A. Proliferación de escuelas de medicina en Latinoamérica: causas y consecuencias. Rev Med Chile. 2002;130(8):917-24.
18. Crotty BJ. More students and less patients: the squeeze on medical teaching resources. Med J Austr. 2005;183(9):444-5.

19. Vukusich A, Larrea R, Alamo M, Ponce JC, Valls G, Guiterrez $\mathbf{M}$, et al. Docencia de pregrado en medicina interna en un hospital privado: diez años de experiencia. Rev Med Chile. 2009;137:1105-12.

20. Sousa AC, Tajra CR, Coelho RS, Gomes CM, Teixeira RA. Medical learning in a private hospital: patients' and companions' perspectives. Sao Paulo Med J. 2009;127(2):101-4.

21. Sayer M, Bowman D, Evans D, Wessier A, Wood D. Use of patients in professional medical examinations: current UK practice and the ethicolegal implications for medical education. BMJ. 2002;324:404-7.

22. Torres-Noriega J. Los exámenes nacionales de medicina (ENAM) en el Perú. Rev Peru MEd Exp Salud Publica. 2008;25(3):316-8.

23. Huamaní C, Gutierrez C, Mezones-Holguín E. Correlación y concordancia entre el examen nacional de medicina y el promedio ponderado universitario: análisis de la experiencia peruana en el periodo 2007-2009. Rev Peru Med Exp Salud Publica. 2011;28(1):62-71.

Correspondencia: Dr. Javier Alva

Dirección: Emilio Fernández 356/404. Santa Beatriz, Lima 1, Perú.

Teléfono: (511) 999-127040

Correo electrónico: jag10pe@gmail.com

\section{Consulte las ediciones anteriores de la Revista Peruana de Medicina Experimental y Salud Pública en}

\section{wWw.scielosp.org}

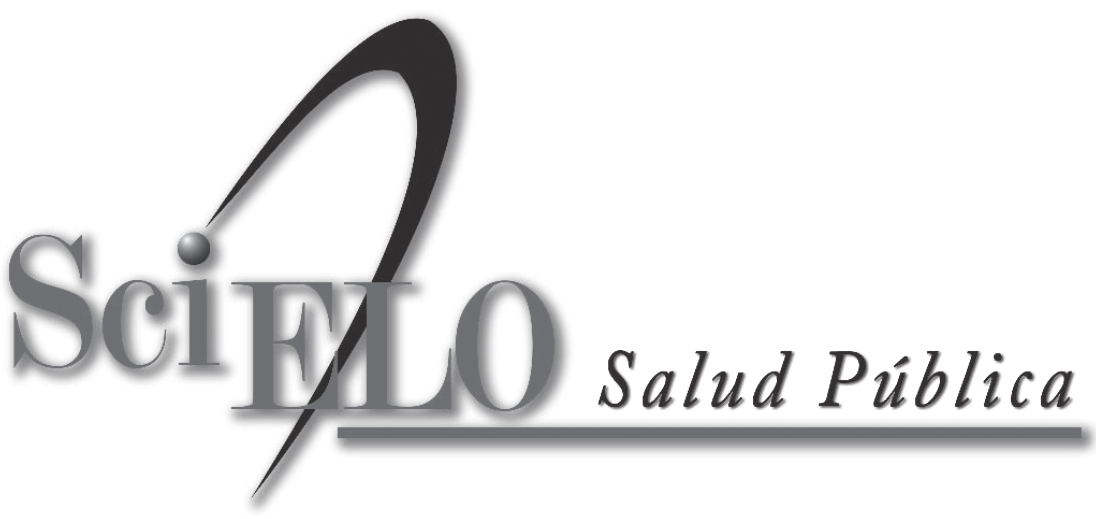

